\title{
Comunidad de aprendizaje con el uso de MOOC sobre CSC en la formación permanente de profesores
}

\author{
María Alejandra Beltrán Penagos \\ Leonardo Fabio Martínez Pérez ${ }^{2}$ \\ Jorge Enrique Ramírez Martínez ${ }^{3}$
}

Recibido: 20-02-2017

Aceptado: 23-04-2017

\section{RESUMEN}

Se presenta la sistematización de la experiencia piloto de un MOOC en el que participaron 38 profesores en diferentes niveles de formación con el fin de que aborden las Cuestiones Sociocientíficas (CSC) como estrategia didáctica en sus prácticas pedagógicas. La sistematización se desarrolla según los planteamientos de Mejía (2012), haciendo una categorización de cinco aspectos de análisis transversales: práctica pedagógica/actualización profesional; estrategias/recursos de enseñanza-aprendizaje; abordaje de CSC; contexto/cotidianidad; y formación ciudadana. Para la discusión de los resultados, se parte de la interpretación de la información elaborada por los profesores participantes durante el desarrollo del MOOC y del conocimiento de la literatura que se trae a la indagación. Se encontraron debilidades en cuanto a la plataforma, a las habilidades tecnológicas, a la autonomía, a la comunicación profesor-estudiante, y a la evaluación. Las oportunidades analizadas se refieren a los espacios virtuales para la enseñanza-aprendizaje y a los momentos de participación. En cuanto

1. Mg (c) María Alejandra Beltrán Penagos es profesora e investigadora de la Universidad Nacional de Colombia [Bogotá], becaria Jóvenes Investigadores (COLCIENCIAS)

Correo: mbeltranp@unal.edu.co-male.belp@gmail.com ORCID: https://orcid.org/0000-0003-4546-8224

2. Dr. Leonardo Fabio Martínez Pérez es profesor de la Facultad de Ciencia y Tecnología de la Universidad Pedagógica Nacional, Doctor Educação Para a Ciência Universidade Estadual Paulista Julio de Mesquita Filho.

Correo: lemartinez@pedagogica.edu.co

ORCID: https://orcid.org/0000-0001-8884-8847

3. Mg. Jorge Enrique Ramírez Martínez es profesor e investigador de la Universidad Santo Tomás [Bogotá].

Correo: jorgeramirezm@ustadistancia.edu.co - kikeupn@gmail.com

ORCID: https://orcid.org/0000-0002-1171-9710 
a las fortalezas, se evidenciaron respecto a un escenario virtual, a la secuencialidad y al rol de los estudiantes. Finalmente, se analizan algunas de las amenazas de la experiencia a nivel general en cuanto a la adaptación a una sociedad en red y al uso de las redes sociales en educación.

Palabras clave: MOOC, CSC, formación de profesores, sistematización de experiencias, TIC.

\title{
Community of learning with the use of MOOC on CSC in the permanent formation of professors
}

\begin{abstract}
It is presented the systematization of the pilot experience of a MOOC in which 38 teachers participated in different training levels in order to address the Socio-Scientific Questions (CSC) as a didactic strategy in their pedagogical practices. The systematization is developed according to Mejía (2012), making a categorization of five aspects of transversal analysis: pedagogical practice / professional updating; strategies / teachinglearning resources; CSC approach; context / everydayness; and citizen education. For the discussion of the results, it starts from the interpretation of the information elaborated by the participating professors during the development of the MOOC and of the knowledge of the literature that is brought to the inquiry. Weaknesses were found regarding the platform, technological skills, autonomy, teacher-student communication, and evaluation. The opportunities analyzed make reference to virtual spaces for teaching-learning and the moments of participation. As for the strengths, they were evident in a virtual scenario, sequentiality and the role of students. Finally, some of the threats of the experience at a general level are analyzed in terms of adaptation to a networked society and the use of social networks in education.
\end{abstract}

Keywords: MOOC, CSC, teachers' training, systematization of experiences, ICT.

Descriptores: Movimiento educativo abierto, Prácticas formativas abiertas, Redes sociales y entornos virtuales de aprendizaje en educación 


\section{Introducción}

Con el fin de que los profesores desarrollaran estrategias didácticas desde el abordaje de las cuestiones sociocientíficas en el aula de clase con la participación en una comunidad de aprendizaje; se realizó una propuesta de MOOC (Masive Open Online Course) denominado Cuestiones Socio Científicas (CSC) como estrategia didáctica. En el desarrollo de este curso se desarrollaron seis fases que se mencionan en una primera entrega; en la que específicamente se desarrollan las de planteamiento general del MOOC, de planificación y de diseño de materiales. Las fases de sistematización y la de evaluación y análisis de los resultados, se desarrollan a continuación.

Para el ejercicio de investigación y análisis, se emplean cuestionarios de inicio y de cierre como herramientas de recolección de información en cuanto a las características generales de la población y de valoración del curso. A su vez, las participaciones en las actividades propuestas del curso son analizadas e interpretadas con el fin de conocer los aportes y las dificultades que se presentan en el abordaje de las cuestiones sociocientíficas discutidas en un ambiente virtual en la formación continuada de profesores (Viejo, Cabezas, \& Martínez, 2013).

Fomentando el análisis cualitativo, se construyen algunas categorías conceptuales, sus propiedades (subcategorías y aspectos significativos de las categorías) e hipótesis (relaciones entre ellas) en el marco del Método de Comparación Constante (Glaser y Strauss, 1967 citado por Valles, 1999) y para ello se realiza una categorización o codificación inicial de los datos brutos con el fin de abrir la indagación.

Este ejercicio de sistematización e interpretación se realiza desde el inicio del desarrollo del MOOC y no al final, ya que como lo menciona Mejía (2012), cuando se tiene la sistematización desde los resultados y no desde el proceso, se tiende a "modelizar", a perder de vista el proceso vivido y se termina dando una mirada sobre el proyecto realizado. Lo que trae como peligro el no dar cuenta de las modificaciones que se establecen en el camino y hacer una reconstrucción desde las concepciones teóricas, o tender a "positivizar" el resultado de la experiencia, perdiendo la riqueza subjetiva que se genera en el proceso (de la Calle et al., 2014). 


\section{Participantes de la investigación}

Para el desarrollo de la experiencia piloto del MOOC se inscribieron un total de 38 participantes de la ciudad de Bogotá, Colombia. La formalización de la inscripción al MOOC, se lleva a cabo con un cuestionario de caracterización inicial, que es diseñado como instrumento de estudio diagnóstico para conocer los perfiles de los participantes del curso ${ }^{4}$, de los cuales 14 tienen títulos de maestría, 6 son estudiantes de maestría, 2 son estudiantes de pregrado y 16 son estudiantes normalistas pertenecientes a la Escuela Normal Superior María Auxiliadora.

A partir de esta herramienta diagnóstica, se identifica que en el grupo de participantes prima el rango de edad entre 18 - 25 años, presentándose personas con edades hasta el rango de 56 - 65 años. Los profesores participantes son mayormente del área de ciencias, sin embargo en la población se encuentran también profesores de humanidades, tecnología y artes, prevaleciendo los profesores de secundaria. En cuanto a los años de experiencia, predominan los profesores que no tienen años de experiencia (normalistas). En la tabla 1 se muestran algunas de sus características:

4. https://www.e-encuesta.com/r/IxAmNDlcVJt2Y3ctwWkHLw/

Tabla 1. Caracterización de los participantes del MOOC

\begin{tabular}{|c|c|c|}
\hline Aspectos & Características & Respuestas \\
\hline \multirow{4}{*}{ Información personal } & Nombre & 36 respuestas totales \\
\hline & Género & $\begin{array}{l}\text { Femenino } 86,11 \% \text { y Masculino } \\
13,89 \%\end{array}$ \\
\hline & Edad & $\begin{array}{l}17 \text { de } 18-25 \text { años } \\
7 \text { de } 26-35 \text { años } \\
5 \text { de } 36-40 \text { años } \\
2 \text { de } 41-45 \text { años } \\
2 \text { de } 46-50 \text { años } \\
1 \text { de } 51-55 \text { años } \\
2 \text { de } 56 \text { a } 65 \text { años }\end{array}$ \\
\hline & Nivel académico máximo alcanzado & $\begin{array}{c}\text { Master } 41,67 \% \text {; Secundaria } 36,11 \% \\
\text { y Pregrado } 22,22 \%\end{array}$ \\
\hline \multirow{3}{*}{ Cursos en línea } & Experiencia con cursos en línea & $\begin{array}{l}\text { Sí } 58,33 \% \\
\text { No } 41,67 \%\end{array}$ \\
\hline & Tipo de curso & $\begin{array}{l}\text { Otro } 47,22 \% \text {; Curso para formación } \\
\text { laboral } 27,78 \% \text {; Curso basado en } \\
\text { recursos de acceso abierto } 8,33 \% \text {; } \\
\text { Curso para créditos universitarios } \\
\text { 8,33\%; Otro MOOC } 8,33 \%\end{array}$ \\
\hline & $\begin{array}{l}\text { Experiencias de aprendizaje } \\
\text { colaborativo en los cursos en línea }\end{array}$ & $\begin{array}{l}\text { Si } 63,89 \% \\
\text { No } 36,11 \%\end{array}$ \\
\hline
\end{tabular}




\begin{tabular}{|c|c|c|}
\hline \multirow{3}{*}{ Experiencia profesional } & Nivel en el que ejerce & $\begin{array}{c}\text { Profesor de primaria } 27,78 \% \\
\text { Profesor de secundaria } 47,22 \% \\
\text { Profesor universitario } 8,33 \% \\
\text { Profesor no formal } 8,33 \% \\
\text { No soy profesor } 8,33 \%\end{array}$ \\
\hline & Tiempo de experiencia & $\begin{array}{c}5 \text { respuestas de más de } 20 \text { años } \\
7 \text { respuestas de } 10 \text { a } 15 \text { años } \\
4 \text { respuestas de } 5 \text { a } 10 \text { años } \\
8 \text { respuestas de } 1 \text { a } 5 \text { años } \\
3 \text { respuestas de ninguna }\end{array}$ \\
\hline & Área de enseñanza & $\begin{array}{c}\text { Ciencias naturales } 12 \\
\text { Química } 5 \\
\text { Todas las asignaturas } 4 \\
\text { Ninguna 3 } \\
\text { Matemáticas } 3 \\
\text { Inglés/español } 2 \\
\text { Ciencias sociales } 2 \\
\text { Tecnología e informática } 1 \\
\text { Sociales } 1 \\
\text { Artes 1 } \\
\text { Educación } 1\end{array}$ \\
\hline \multirow{4}{*}{$\begin{array}{l}\text { Aproximaciones a las } \\
\text { CSC }\end{array}$} & $\begin{array}{l}\text { ¿Los aspectos científicos, } \\
\text { ambientales, económicos y sociales } \\
\text { que le generan controversia son } \\
\text { propicios para el proceso de } \\
\text { enseñanza - aprendizaje en su } \\
\text { clase? }\end{array}$ & $\begin{array}{c}\text { A menudo } 44,44 \% \\
\text { Casi siempre } 30,56 \% \\
\text { Algunas veces } 19,44 \% \\
\text { Pocas veces } 5,56 \%\end{array}$ \\
\hline & $\begin{array}{c}\text { ¿Desarrolla habilidades en su clase } \\
\text { incluyendo estrategias educativas } \\
\text { de tipo multidisciplinar? }\end{array}$ & $\begin{array}{c}\text { A menudo } 38.89 \% \\
\text { Casi siempre } 33,33 \% \\
\text { Algunas veces } 22,22 \% \\
\text { Pocas veces } 5,56 \%\end{array}$ \\
\hline & $\begin{array}{l}\text { ¿En su práctica involucra el } \\
\text { manejo de problemáticas vigentes } \\
\text { que relacionen la enseñanza de } \\
\text { su asignatura con la formación } \\
\text { ciudadana? }\end{array}$ & $\begin{array}{c}\text { A menudo } 41,67 \% \\
\text { Casi siempre } 38,89 \% \\
\text { Pocas veces } 13,89 \% \\
\text { Algunas veces } 5,56 \%\end{array}$ \\
\hline & $\begin{array}{c}\text { ¿Ha trabajado con Cuestiones } \\
\text { Sociocientíficas en la enseñanza de } \\
\text { su asignatura? }\end{array}$ & $\begin{array}{l}\text { Algunas veces } 50 \% \\
\text { A menudo } 19,44 \% \\
\text { Nunca } 11,11 \% \\
\text { Pocas veces } 11,11 \% \\
\text { Casi siempre } 8,33 \%\end{array}$ \\
\hline \multirow{2}{*}{$\begin{array}{c}\text { Experiencias de } \\
\text { enseñanza-aprendizaje }\end{array}$} & $\begin{array}{c}\text { ¿Establece una búsqueda } \\
\text { permanente de estrategias para } \\
\text { fomentar la educación investigativa } \\
\text { y de contexto? }\end{array}$ & $\begin{array}{c}\text { A menudo } 38.89 \% \\
\text { Algunas veces } 27,78 \% \\
\text { Casi siempre } 25 \% \\
\text { Pocas veces } 8,33 \%\end{array}$ \\
\hline & $\begin{array}{l}\text { ¿Desarrolla trabajo entre pares } \\
\text { que posibilite el intercambio de } \\
\text { opiniones y puntos de vista, } \\
\text { logrando así, una reflexión común? }\end{array}$ & $\begin{array}{l}\text { Casi siempre } 33,33 \% \\
\text { Algunas veces } 27,78 \% \\
\text { A menudo } 19,44 \% \\
\text { Pocas veces } 19,44 \%\end{array}$ \\
\hline
\end{tabular}

Fuente. Adaptada de (Alemán, 2015)

A pesar de que inicialmente se pensó que la mayoría de participantes serían profesores en ejercicio, se adaptaron algunas actividades debido a la influencia del público participante perteneciente a la Escuela Normal Superior María Auxiliadora. 


\section{Discusión y análisis}

Desde los planteamientos de sistematización realizados por Mejía (2012), se realiza una lectura e interpretación inicial de las actividades y comentarios de los participantes del MOOC, que permite evidenciar cinco aspectos de análisis transversales que están presentes en los diferentes momentos y participaciones del curso y que le dan una unidad al mismo en términos de aportes y dificultades: práctica pedagógica y actualización profesional, estrategias y recursos de enseñanza-aprendizaje, abordaje de CSC, contexto y cotidianidad, y formación ciudadana (Pérez, 2013). Estos aspectos son concretados mediante la evidencia de las afirmaciones mostradas en los siguientes apartados, y que se relacionan con las participaciones de los estudiantes y la actividad donde se hizo evidente.

Para el proceso de sistematización, se tuvo en cuenta la formación académica de cada participante para relacionar sus intervenciones en el curso con las convenciones que se muestran en la tabla 2, teniendo en cuenta que participaron 16 estudiantes normalistas (Nor), 6 estudiantes de maestría (EM) y 14 profesores con maestría (M).

Tabla 2. Caracterización de los participantes del MOOC.

\begin{tabular}{|c|c|}
\hline Formación académica del participante & Convención \\
\hline Estudiante normalista & Nor (1-16) \\
\hline Estudiante de maestría & EM (1-6) \\
\hline Master & $M(1-14)$ \\
\hline
\end{tabular}

Fuente. Elaboración propia.

En la tabla 3 se relacionan las convenciones que hacen referencia a las actividades que desarrollaron los participantes en las que se evidenciaron los respectivos aportes y dificultades durante el desarrollo de las actividades y las participaciones del MOOC, lo cual permite darle sentido a los momentos y actores en los que se presentan.

Tabla 3. Convenciones de las actividades del MOOC.

\begin{tabular}{|c|c|}
\hline $\begin{array}{c}\text { Participación donde se hizo } \\
\text { evidente el aspecto de análisis }\end{array}$ & Convención \\
\hline Actividad 1 & $\bullet$ \\
\hline P2P N 1 & v \\
\hline P2P N 2 & ü \\
\hline
\end{tabular}

\begin{tabular}{|c|c|}
\hline P2P N³ & $\varnothing$ \\
\hline Participaciones & 0 \\
\hline
\end{tabular}

Fuente. Elaboración propia. 


\section{Práctica pedagógica / Actualización profesional}

El aspecto de práctica pedagógica y actualización profesional constituyó un pilar importante porque de acuerdo con el perfil de los participantes y a sus expectativas e intereses con respecto al $\mathrm{MOOC}$, mencionaron el mejorar y actualizar su práctica pedagógica, por otra parte el buscar, conocer, crear y desarrollar nuevas estrategias que favorezcan no solamente la enseñanzaaprendizaje de las ciencias sino de variados escenarios, abordando las CSC como un medio de motivación para liderar procesos de cambio con responsabilidad social (Azevedo, 2014).

El MOOC se reconoce como un espacio para fortalecer el aprendizaje motivado autónomamente, de forma crítica, para validar desde la experiencia lo propuesto y para que a partir de estas experiencias y de sus conocimientos, se pueda construir colaborativamente y poner en práctica lo aprendido de acuerdo con cada contexto (Pérez, 2014).

En la tabla 4 se evidencian algunos apartes de los participantes (ver tabla 2) tomados de las actividades que se desarrollaron en el curso (relacionadas en la tabla 3) en los que se evidencian aportes y dificultades relacionadas con el aspecto de práctica pedagógica y actualización profesional.

Tabla 4. Aportes y dificultades en el aspecto de práctica pedagógica y actualización profesional.

\begin{tabular}{|c|c|}
\hline Aportes & Dificultades \\
\hline $\begin{array}{c}\text { Expectativas por mejorar la práctica } \\
\text { pedagógica. (A1) }\end{array}$ & $\begin{array}{c}\text { ü Las preguntas orientadoras formuladas son } \\
\text { cerradas, de definición o explicación causal (M1, } \\
\text { EM2, Nor12, Nor5). } \\
\text { ü Las intenciones del profesor no son claras, } \\
\text { se reducen al uso de un recurso o a la reflexión } \\
\text { en torno a un tema (ruiz-Nor14), o a abordar } \\
\text { las CSC para trabajar con los estudiantes de } \\
\text { manera más didáctica y amena (Mora-Nor6). } \\
\varnothing \text { Se evidencia falta de intencionalidad de } \\
\text { los participantes en el desarrollo del P2P N³3 } \\
\text { (Nor14) (Nor5) (Nor6) (Nor2) (Nor16) (Nor7) } \\
\varnothing \text { Incidencia de aspectos tradicionales en la } \\
\text { evaluación (Nor3) }\end{array}$ \\
\hline
\end{tabular}

Fuente. Elaboración propia. 
A la hora de proponer actividades de clase, los participantes propusieron algunas preguntas orientadoras para la el abordaje de las CSC y se evidenció que varias de estas preguntas son cerradas, de definición o explicación causal. Por ejemplo, la profesora EM5 propuso la CSC a partir de la pregunta cerrada "¿Qué es más importante para mi ciudad: su naturaleza o sus construcciones?" y para abordarla formula otras preguntas como: “Qué son los humedales?, ¿Cuáles son los humedales de Bogotá?, ¿Qué especies de fauna y flora habitan en los humedales?, ¿Por qué son importantes los humedales?, ¿Qué acciones del hombre afectan a los humedales? (...)". Frente a lo anterior, en el proceso de heteroevaluación, se sugirió a las profesoras el planteamiento de algunas preguntas orientadoras no solo para que los estudiantes definan o expliquen la causa de algo, sino de otra variedad que implique más allá de una consulta o una respuesta corta (Konieczny, 2015). Como por ejemplo preguntas que inviten a la predicción: ¿Qué pasaría si...? la comprobación: ¿Cómo se puede saber...? la gestión: ¿Qué se puede hacer...? ¿Cómo se podría hacer...? o la opinión: ¿Qué piensas...? (Manjarrés, Mejía, Bravo, Boada y Jiménez, 2007).

Respecto a las dificultades en este aspecto de las prácticas pedagógicas, se evidenció que en varios casos las intenciones del profesor no son claras o se reducen al uso de un recurso para la reflexión en torno a un tema (Nor14) o a abordar las CSC para trabajar con los estudiantes de manera más didáctica y amena (Nor6), sin considerar un objetivo de enseñanza específico sobre el abordaje de las CSC (Martínez y Pacheco, 2012). En el planteamiento de las actividades acordes con la CSC elegida, es importante que exista claridad en las intenciones y finalidades que se pretenden con ellas, asi como es necesario explicar cuál es el aporte que tendrá la CSC trabajada (por medio de las actividades planteadas) para la formación del estudiante.

Por otra parte en la planeación de la metodología y los parámetros de evaluación que construyeron los participantes para desarrollar en su experiencia de aula de acuerdo con la CSC elegida, se hace evidente la incidencia de aspectos tradicionales, "Se tomará en cuenta puntualidad, participación en clase, manejo de autores frente a conceptos propios de la temática" (Nor3), dejando de lado la apropiación de habilidades como la argumentación, la claridad frente al tema o analizar la información.

\section{Estrategias / recursos de enseñanza-aprendizaje}

Es considerado un aporte que los participantes del MOOC busquen conocer, crear y desarrollar nuevas estrategias para favorecer el aprendizaje de sus 
estudiantes, ya que esto implica el desarrollo de diferentes reflexiones y acciones para animar su motivación y posibilitar el desarrollo de algunas habilidades de pensamiento crítico en los estudiantes (Martínez y Pacheco, 2012) que se manifiestan por un aprecio por el trabajo con CSC.

De acuerdo con las respuestas dadas por los participantes con la propuesta de las actividades en la $\mathrm{P} 2 \mathrm{P} \mathrm{N}^{\circ} 3$, se evidencia la realización secuencial de las actividades con respecto a la complejidad de la controversia elegida, por ejemplo las profesoras Nor14, EM5, Nor5, M1 y EM1 trabajaban desde una actividad de indagación sobre la información que tienen los estudiantes (lluvia de ideas), hasta la realización de la reflexión escrita sobre la información adquirida en el proceso.

Cuando se pretende abordar una CSC en el aula es necesario pensar el diseño curricular de manera propicia para tal fin, por ende al participante presentarlo con los contenidos curriculares y la apropiación de la actividad significa una planeación clara sobre la apropiación de la CSC en el aula (Ferreyra, 2014). Y aunque varios maestros pusieran en evidencia la controversia y el objetivo de su abordaje como CSC, no se presentaba claridad en cuanto a la integración curricular de la misma.

Uno de los planteamientos de Zeidler (2003) es la importancia de entender el papel de la ciencia con otras áreas de la vida, conectando su abordaje directamente con su incidencia social, ambiental, tecnológica y ético-moral. Evidencia de esto es lo escrito por la profesora Nor16 quien reflexiona sobre la controversia "como situación social" tratándola "desde la interdisciplinaridad de las áreas para ayudar a los estudiantes en la visión de una realidad". Como ella, la posición de los participantes es la implicación de la CSC elegida en diferentes áreas del conocimiento, en las actividades se evidencia que se espera lograr transdisciplinaridad al integrarlas en el aula. Un ejemplo de ello es lo descrito por la profesora Nor1 quien plantea que para llegar a las "posibles soluciones o conclusiones, que den respuesta a la cuestión que se está desarrollando, se trabajarán distintas áreas del conocimiento". 
Tabla 5. Aportes y dificultades en el aspecto de estrategias/recursos de enseñanza-aprendizaje.

\begin{tabular}{|c|c|}
\hline Aportes & Dificultades \\
\hline $\begin{array}{c}\text { Buscar, conocer, crear y desarrollar nuevas } \\
\text { estrategias para favorecer el aprendizaje }\end{array}$ & \\
$\varnothing$ Las actividades planteadas en la P2P N³ \\
$\begin{array}{c}\text { se presentan secuencialmente con respecto al } \\
\text { abordaje de la controversia (Nor14) (EM5) (Nor5) } \\
\text { (M1) (EM1) }\end{array}$ & $\begin{array}{c}\text { ü Se confunde la estrategia didáctica con el } \\
\text { recurso didáctico (Nor12) }\end{array}$ \\
$\begin{array}{c}\varnothing \text { Al presentar las actividades elegidas para } \\
\text { abordar la CSC, se muestra una aproximación a } \\
\text { un diseño curricular (EM5) (Nor1) (M1) (EM1) }\end{array}$ & $\begin{array}{c}\varnothing \text { Al elegir un tema, algunas veces no se } \\
\text { plantea la controversia para dar paso a la CSC } \\
\text { que se abordara en el aula, se define solo una } \\
\text { actividad que supone una articulación en el } \\
\text { currículo (Nor6) (Nor2) (Nor16) (Nor7) }\end{array}$ \\
$\begin{array}{c}\varnothing \text { Las actividades propuestas para abordar } \\
\text { las CSC como estrategia didáctica, es la } \\
\text { implicación en diferentes áreas del conocimiento: } \\
\text { transdisciplinaridad. (Nor1) (Nor16 }\end{array}$ \\
$\begin{array}{c}\text { o Las CSC válidas para la investigación } \\
\text { definición y aportes de la misma (Nor11) (Nor16) }\end{array}$ \\
\hline
\end{tabular}

Fuente. Elaboración propia.

En otros casos, la estrategia didáctica se hace equivalente al recurso didáctico (Nor12) que se plantea, es decir que se tiene la concepción de que la estrategia didáctica es igual al recurso didáctico (actividad) dejando de lado el proceso amplio y los demás elementos curriculares bajo los que se proponen las estrategias (objetivos, justificación, definición de la CSC, entre otros).

\section{Abordaje de CSC}

El abordaje de CSC es la parte esencial del MOOC, ya que se espera que sea la estrategia didáctica que usen los participantes para la orientación de su práctica pedagógica. En el desarrollo de la actividad P2P №1, se evidenció que en su mayoría, los profesores participantes reconocen las características de una CSC y resulta valioso que la reconozcan como una estrategia para liderar procesos de cambio con responsabilidad social tanto en ellos como en sus estudiantes; así como para estimular la curiosidad y para dar profundidad a las búsquedas de los estudiantes, pues de trata no sólo de que conozcan y absorban la información que el profesor o los medios de comunicación les proporcionan, reproduciendo y legitimando todo un sistema (Aparicio, 2017); sino que por el contrario, tengan la curiosidad para indagar y cuestionar la credibilidad y la veracidad de las fuentes. Acorde con ello, como reto "el docente debe realizar un aumento en su nivel de argumentación junto con el estudiante para desarrollar la habilidad para provocar la argumentación, la habilidad para relacionar el 
lenguaje científico con el lenguaje cotidiano y la habilidad para cualificar el proceso de argumentación" (Martínez y Parga, 2013).

Una observación interesante es la de la profesora EM5, donde identifica las CSC como una oportunidad para el "desarrollo de procesos en dimensiones del ser humano (cognitiva, comunicativa, corporal, socioafectiva)", pues generalmente se habla de los aspectos científicos, críticos y éticos, y se deja de lado este aspecto tan importante, que puede ser un referente para plantear los objetivos de aprendizaje en relación a estas dimensiones.

Tabla 6. Aportes y dificultades en el aspecto de Abordaje de CSC

\begin{tabular}{|c|c|}
\hline Aportes & Dificultades \\
\hline $\begin{array}{l}\text { • Las CSC como un recurso o un medio de } \\
\text { motivación. } \\
\text { • Las CSC para liderar procesos de cambio con } \\
\text { responsabilidad social } \\
\text { v Reconoce las CSC como controversias con } \\
\text { implicaciones sociales (a nivel teórico) } \\
\text { v Reconocen las características de una CSC } \\
\text { v Para estimular la curiosidad } \\
\text { vera el desarrollo de procesos en dimensiones del } \\
\text { ser humano (cognitiva, comunicativa, corporal, socio- } \\
\text { afectiva) (EM5) } \\
\text { Ø Con las actividades hay un fomento a la } \\
\text { investigación vista desde lo que espera cada } \\
\text { participante al implicar las CSC en el aula. (Nor3) } \\
\text { o EM } 1 \text { enfatiza en como las CSC son estrategias } \\
\text { didácticas para la investigación }\end{array}$ & 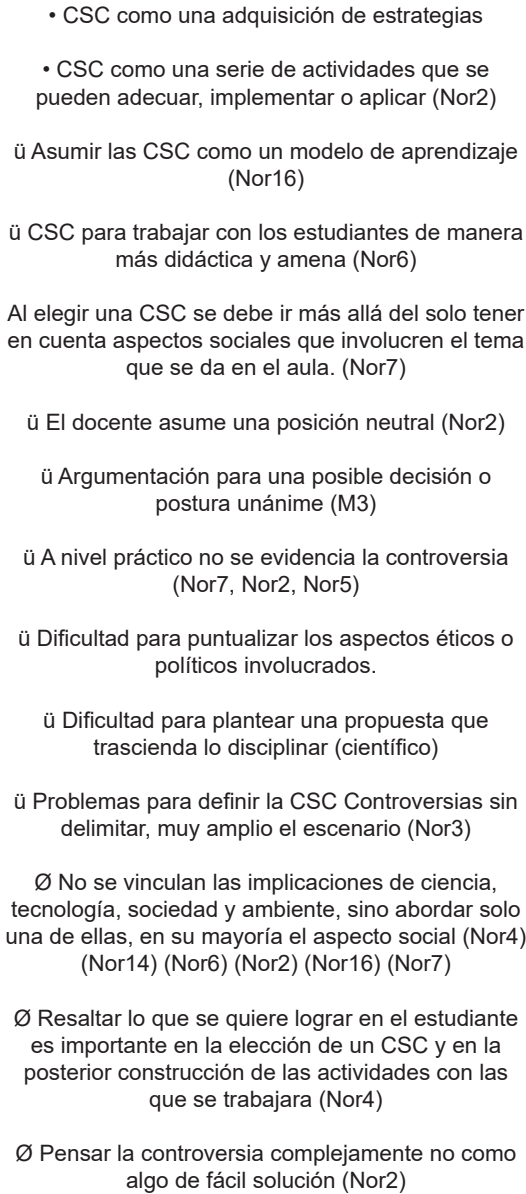 \\
\hline
\end{tabular}

Fuente. Elaboración propia. 
En la tabla 6, se observa que cuatro participantes (Nor11, EM4, Nor12, Nor2) entienden el abordaje de las CSC como una adquisición de estrategias o una serie de actividades que se pueden adecuar, o se asumen las CSC como un "modelo" de aprendizaje, lo cual evidencia dificultades de formación pedagógica para comprender los modelos de aprendizaje, las líneas de investigación en didáctica y la diferenciación entre ellas. Una posible explicación a esta situación puede atribuirse al nivel académico alcanzado, relacionado con la experiencia profesional.

Por otra parte, el querer llegar a adoptar una postura unánime con el grupo con el que se aborda la CSC o asumir una posición "neutral" como profesor evidencia debilidades para lidiar con las situaciones controvertidas, pues como lo señalan Franco y Santos (2015), estas dificultades pueden estar relacionadas con las concepciones epistemológicas de los profesores sobre la naturaleza de la Ciencia, con una comprensión del conocimiento científico como listo y configurado, sin espacio para preguntas y posibilidades de otras opiniones.

Otra característica muy notable, es que a pesar de que en la actividad $\mathrm{P} 2 \mathrm{P} \mathrm{N}^{\circ} 1$ se evidenció un reconocimiento teórico de las características de una CSC, a nivel práctico no se evidenciaron las controversias y se propusieron temáticas muy amplias; fué ahí cuando se realizó un mayor acompañamiento y una propuesta de evento virtual para hacer una retroalimentación a las dudas y propuestas que se dieron en ese momento de la formación.

En cuanto a esta categoría, los participantes mostraron dificultades a la hora de realizar un ejercicio de profundización de las dimensiones involucradas en la CSC, incluso de la misma dimensión científica. En este sentido, como lo sugiere Martínez (2014), uno de los desafíos para la formación del profesorado está en conocer y aprender los distintos aspectos científicos, filosóficos, sociológicos, políticos, ambientales y éticos que contribuyan a fundamentar y orientar su práctica de enseñanza.

\section{Contexto / cotidianidad}

Los participantes reconocen que con el curso MOOC pueden poner en práctica lo aprendido de acuerdo con cada contexto, lo cual ofrece una mejor aplicabilidad y relevancia de la enseñanza-aprendizaje en la vida diaria teniendo en cuenta que como lo proyecta la profesora Nor5: "(...) así el desarrollo de la CSC se llevará a cabo con mayor facilidad e interés por 
parte del alumnado, logrando que el interés de los niños no se apague y el aprendizaje sea para la vida, pues encuentren el sentido e importancia de saber acerca del tema".

Algo que Martínez y Parga (2013) remarcan en la elección de la CSC para abordarla en el aula de clase es que sea una controversia contextualizada, para que el interés en los estudiantes corresponda a lo esperado por el profesor. Los participantes también consideran las CSC válidas para la investigación educativa, teniendo en cuenta que permiten promover una ciencia escolar de mayor validez y utilidad para los estudiantes, y a su vez estimular diferentes aprendizajes como el aprendizaje cooperativo o por indagación.

Tabla 7. Aportes y dificultades en el aspecto de contexto/cotidianidad.

\begin{tabular}{|c|c|}
\hline Aportes & Dificultades \\
\hline $\begin{array}{l}\text { • Poner en práctica lo aprendido de acuerdo con } \\
\text { cada contexto } \\
\text { v Enseñanza en contextos particulares para } \\
\text { mejor aplicabilidad y relevancia en la vida diaria } \\
\text { ü Aprendizaje para la vida (Nor5) } \\
\text { ü Las CSC parten de las necesidades de los } \\
\text { estudiantes como ciudadanos (Nor9) } \\
\varnothing \text { En el planteamiento de las actividades } \\
\text { acordes con la CSC elegida, la contextualización } \\
\text { de la controversia es útil para interesar a los } \\
\text { estudiantes de cada participante (Nor3) } \\
\text { o Contextualización evidencia mayor utilidad en el } \\
\text { conocimiento de los estudiante (Nor3) }\end{array}$ & $\begin{array}{c}\text { ü Problemas para definir la CSC Controversias } \\
\text { sin delimitar, muy amplio el escenario (Nor3) } \\
\text { o Aporte en cuanto a la planeación de clase, } \\
\text { partiendo de un acercamiento a la realidad de } \\
\text { los estudiantes (Nor9) }\end{array}$ \\
\hline
\end{tabular}

Fuente. Elaboración propia.

Es importante tener en cuenta que la toma de decisiones no debe reducirse al desarrollo de estrategias didácticas que contribuyan a la construcción de posicionamientos personales de los estudiantes, sino además debe considerar la forma como el contexto cultural influye en dicho proceso (Reis, 2014). En este sentido se sugirió delimitar o puntualizar algunas de las CSC planteadas porque abarcaban una situación muy general, muy amplia y sin casos concretos de estudio.

\section{Formación ciudadana}

Propiciar la participación y la toma de decisiones son algunas de las principales características de las CSC que son apropiadas en los 
participantes durante el desarrollo de sus actividades. Señala la profesora EM5 que "iniciar con el desarrollo de procesos argumentativos en el campo de las ciencias naturales y empezar a construir ideas propias frente a la responsabilidad social que tenemos como ciudadanos y el cuidado del medio ambiente" son algunas de las acciones que se deben realizar para fortalecer el ejercicio de la ciudadanía.

Abordar las CSC también involucra una cultura de la indagación (Nor1, Nor16) en la que el profesor está en una constante búsqueda de fuentes y oportunidades para generar debates en el aula y para fomentar el pensamiento crítico y la realización de juicios éticos y morales (EM5) en los estudiantes, que se espera incidan sobre su actuar y les permitan asumirse con mayor propiedad como agentes de trasformación social responsables de su futuro (Nor1, Nor9).

Involucrando los aportes de la formación ciudadana la profesora EM5, propone potencializar el desarrollo de las "competencias ciudadanas y éticas en los estudiantes relacionadas con el cuidado del medio ambiente y los valores cívicos", y la profesora Nor3 menciona como objetivo formar "estudiantes con rol protagónico en la construcción de sus opiniones y toma de decisiones en pro de un bienestar social".

Tabla 8. Aportes y dificultades en el aspecto de práctica pedagógica y actualización profesional.

\begin{tabular}{|c|c|}
\hline Aportes & Dificultades \\
\hline $\begin{array}{c}\text { v Propiciar la participación y la toma de decisiones. } \\
\text { EM5). }\end{array}$ & \\
$\begin{array}{c}\text { ü Acciones que se pueden realizar como ciudadanos } \\
\text { (EM5). Procesos de negociación (M3) }\end{array}$ & \\
$\begin{array}{c}\text { ü Involucra una cultura de la indagación (Nor1) (Nor16) } \\
\text { ü Estudiantes como agentes de trasformación social } \\
\text { responsables de su futuro (Nor1, Nor9). Estudiantes } \\
\text { activos frente a problemáticas que les incumben al ser } \\
\text { ciudadanos del mundo (Nor1) }\end{array}$ & ü Formación para futuros ciudadanos (M3). \\
$\begin{array}{c}\text { o Se evidencia el desarrollo de competencias } \\
\text { ciudadanas y éticas por parte de los estudiantes (EM5) }\end{array}$ \\
$\begin{array}{c}\text { o Las CSC contribuyen al proceso de aprendizaje de } \\
\text { los estudiantes, quienes adquieren una formación } \\
\text { ciudadana con la toma de decisiones en pro del } \\
\text { bienestar social (Nor3) }\end{array}$ \\
$\begin{array}{c}\text { o La formación de estudiantes, quienes será futuros } \\
\text { ciudadanos para la toma de decisiones, se basa en sus } \\
\text { aprendizajes (M3) }\end{array}$ \\
\hline
\end{tabular}

Fuente. Elaboración propia. 
La profesora M3 hace referencia a una "formación para los futuros ciudadanos" y con relación a este aspecto, en el proyecto "We Act" expuesto por Reis (2014) que tiene la intención de promover las acciones colectivas a partir de las Controversias Socio-Científicas, los estudiantes son reconocidos como agentes importantes de cambio capaces de llevar a cabo acciones y, en consecuencia como "ciudadanos" (en contraposición a una concepción de "ciudadanos del futuro"). De acuerdo con lo anterior se coincide con Invernizzi y Williams (2009), citado por Reis (2014) quienes plantean que los niños y jóvenes deben ser percibidos como actores sociales de pleno derecho ("ciudadanos ahora") y no como ciudadanos en preparación.

\section{Instrumento de análisis tipo DOFA}

Los datos analizados al final del MOOC fueron recopilados con un segundo cuestionario ${ }^{5}$ con el fin de obtener información sobre la experiencia en el curso y caracterizar las debilidades, oportunidades, fortalezas y amenazas presentadas. Para la elaboración de este instrumento se tuvo en cuenta un formato de valoración del syllabus con el fin de contrastar las valoraciones de los pares expertos, con la experiencia propia de los participantes. En la primera parte se indagó sobre los elementos curriculares, de planeación y de desarrollo del curso (extensión del curso, contenidos, actividades, tiempo de dedicación) y la segunda parte consistió en un cuestionario de escala tipo Likert para identificar la comprensión de los estudiantes acerca de las CSC.

\section{Debilidades}

En cuanto a la usabilidad de la plataforma, la profesora M1 menciona: "a mi parecer se observa algo desordenada, en algunas partes se confunden los comentarios de los participantes, no quedan registrados los comentarios de estos participantes en orden de participación y en una misma zona como sí sucede en los foros de moodle". Esto es debido a que la comunidad de G+ tiene un formato de red social, por lo que al hacer anuncios o comentarios, estos se visualizan en el orden en que se publican. Esta plataforma da la opción de organizar los módulos por hipervínculos, sin embargo los participantes no se guiaban por este menú, sino por las publicaciones o participaciones recientes. Esto tiene incidencia en los requerimientos para la participación en MOOCs, pues las habilidades digitales y el uso de TIC 
son importantes para que el manejo de la plataforma no sea un obstáculo o impedimento para el aprendizaje.

Frente a las debilidades presentes en los participantes, se evidenció una de las dificultades a las que hacen referencia Clarà y Barberà (2013) en cuanto al alto nivel de aprendizaje autorregulado y la autonomía que se requiere por parte de los usuarios. Pues como lo enfatiza la profesora Nor16, el MOOC "es un espacio para el auto-aprendizaje, en el conocimiento de nuevos temas que me permiten interpelarme y realizar un reconocimiento más certero acerca de lo que quiero transmitir como docente". Esta autonomía no siempre se evidencia, ya que varios de los participantes al no sentirse regulados por un profesor simplemente abandonan el espacio virtual.

Acorde con lo mencionado con Cabero y Llorente (2014), dos de los grandes problemas del MOOC fueron la tasa de abandono de los estudios por los estudiantes y su baja participación. Sin embargo se considera que las cifras de deserción en los MOOC irán disminuyendo a medida que las personas se empapen de la cultura del aprendizaje digital y el compromiso que ello conlleva, teniendo en cuenta que son los mismos participantes los que realizan la inscripción.

Reconociendo lo anterior, Hill (2013) presenta una tipología diversa de estudiantes en esta modalidad de cursos:

1. Los no participantes, que son aquellos que se inscriben pero después no hacen nada más fuera de registrarse;

2. los observadores o mirones como también se les denomina, que forman el grueso de los participantes y que se dedican a entrar de vez en cuando en el entorno, revisar algunos de los materiales que se ofrecen en el mismo y simplemente a observar lo que está ocurriendo;

3. los participantes pasivos o merodeadores, que lo conforman el grupo de estudiantes que se limitan a ver los vídeos y realizar algún que otro test, sin implicarse en todas las posibles actividades que se llevan a cabo dentro del MOOC (blogs, foros, acciones de trabajo colaborativo,...); y

4. los participantes activos, que conforman el grupo de los alumnos totalmente comprometidos con el curso, que 
participan en todas y cada una de las actividades propuestas por el equipo docente, intentando aprovechar al máximo la experiencia de este nuevo tipo de aprendizaje.

A diferencia de otras investigaciones como la de Kulkarni, PangWei, Chia, Papadopoulos, Koller y Klemeer (2013), citada por Sánchez y Prendes (2015) donde indican que las calificaciones dadas por un grupo de profesores orientadores no difieren significativamente de la dada por los estudiantes a través de la evaluación por pares, en este caso la evaluación si difirió bastante. Probablemente debido a que en la distribución de las asignaciones a valorar, se encontraban cruces entre estudiantes que comparten clases fuera de la virtualidad (en su mayoría normalistas) y optaron por dar valoraciones altas y comentarios en su mayoría positivos a sus compañeros sin justificar sus reflexiones, lo cual puede limitar la perspectiva de los diversos beneficios derivados de la experiencia de aprendizaje que se produce al momento de evaluar (Sánchez y Prendes, 2015).

\section{Oportunidades}

Las oportunidades reflejadas en el MOOC se orientan en el uso de plataformas libres, o redes sociales para la enseñanza-aprendizaje en la formación continuada de profesores. Los espacios virtuales no solo son considerados como contextos a distancia que integran información de manera diferente o entretenida, son también la oportunidad de establecer redes con personas ajenas a su contexto (Liu y otros, 2014) integrando así el aprendizaje colaborativo para fortalecer la práctica pedagógica. La profesora Nor11 aportóo sobre el conectivismo que "permite adquirir información mediante herramientas tecnológicas, las cuales son importantes para alcanzar conocimiento en el cual exista un aprendizaje colaborativo para aprender de experiencias novedosas que nos ayuden a fortalecer nuestra labor docente".

Los videos realizados por los profesores investigadores que fueron los recursos virtuales en el $\mathrm{MOOC}$, significaron una aproximación útil para el uso de CSC como estrategia didáctica a partir de las experiencias de los maestros.

En cuanto a las oportunidades mencionadas en el cuestionario de evaluación, la profesora EM1 promueve al MOOC como "una excelente herramienta virtual para compartir experiencias de aula. La continuidad de 
estrategias como ésta puede brindar la apertura de nuevas comunidades académicas y son importantes para la investigación didáctica. La temática es interesante y novedosa, fue fácil de entender por la coherencia de las actividades". Una oportunidad evidenciada en la profesora Nor15 es que a pesar de "no terminar el curso, fue de gran aporte para" su "crecimiento personal y como maestra en formación", siendo "de gran satisfacción y utilidad que se sigan presentando cursos como estos". Mostrando la participación de diferentes profesores oportuna para el trabajo colaborativo la profesora EM3 dice: "El curso resultó muy interesante, especialmente porque participaron profesores en diferentes momentos de formación lo cual permitió interactuar y aprender de los demás. En general me pareció genial que fuera en línea, pues se evidencia que se pueden lograr grandes cosas a nivel de educación en la plataforma".

\section{Fortalezas}

El MOOC fue percibido como un escenario innovador que representa una forma diferente de enseñanza-aprendizaje, teniendo en cuenta que de acuerdo con el participante M4 desde un inicio lo considera un "espacio para validar desde la experiencia lo propuesto" dado que se desarrolló bajo un enfoque teórico-práctico en el que las actividades estaban diseñadas para que los profesores generaran reflexiones a partir de su experiencia y su contexto de práctica.

Respecto a los elementos curriculares, el 73\% de los participantes que diligenciaron el cuestionario final, calificaron la extensión del curso como adecuada, el apoyo recibido por el docente es calificado como excelente o bueno, así como los recursos educativos proporcionados (vídeos y publicaciones). También se consideró que las actividades fueron claras y congruentes las unas con otras, en palabras de la profesora Nor2: "Durante el desarrollo de los trabajos realizados en la plataforma, se evidencio la secuencia lógica y ordenada de las actividades creando así un proceso de aprendizaje significativo. También se evidencio el acompañamiento de los docentes encargados de la plataforma sin importar que no se estuviera de forma personal, pues se aclaraban dudas, se daban aportes a los trabajos realizados y se realizan distinciones en los ejercicios propuestos".

De acuerdo a lo que se menciona anteriormente, el MOOC sitúa al estudiante en un papel de mayor protagonismo, dado que es él mismo quien controla y administra los momentos y los recursos que son de su interés para la construcción de su aprendizaje, la participante Nor16 
dijo que "es un espacio para el auto-aprendizaje, en el conocimiento de nuevos temas que me permiten interpelarme y realizar un reconocimiento más certero acerca de lo que quiero transmitir como docente". Además como señala la participante Nor3 "la plataforma de actividades del MOOC para abordar cuestiones sociocientíficas fue bastante útil para acceder a nuevos conocimientos y emplear una estrategia en las ciencias que coloca al estudiante en un rol más activo, evaluando su capacidad de análisis y comprensión frente a temáticas de interés común", ya que cuando se planteó el uso de estrategias de evaluación que dan al estudiante un papel activo y la colaboración en línea entre estudiantes con las valoraciones de las actividades P2P, se da una re-definición de los roles del profesor, propiciando también la comunicación bidireccional con los profesores orientadores mediante los eventos y las heteroevaluaciones.

\section{Amenazas}

Los estudiantes con menor actividad en el MOOC corresponden a los profesores de un rango de edad mayor a 30 años. Esto puede indicar una escasa formación en el uso de TIC y además, que estos profesores posiblemente no practiquen el aprendizaje a distancia ni el diseño y desarrollo de acciones formativas online con sus estudiantes. En este sentido, como lo menciona Valverde (2014), hay una "necesidad de adaptación de las metodologías docentes a las características de una sociedad en red". Sin embargo, con el grupo de estudiantes en su mayoría normalistas o de un rango de edad entre los 18 y los 30 años, esta experiencia de formación puede adquirir un significado en términos de lo que conlleva asumir un papel protagónico en la construcción de su aprendizaje desde la virtualidad.

Por otra parte, como en las prácticas pedagógicas el tiempo generalmente es una amenaza, en este proyecto y en la virtualidad también lo fue. A pesar de que en el MOOC el acceso a los contenidos y recursos permanece abierto, en esta prueba piloto los momentos para las valoraciones, retroalimentaciones y eventos virtuales tuvieron un inicio y un fin. En consecuencia, como sugiere la participante EM5, "sería importante ampliar el MOOC en términos de tiempo" considerando también que dentro de las razones para no terminar el curso, la de mayor porcentaje $(36,36 \%)$ corresponde a que el participante pensó que tendría tiempo, pero no lo tuvo; lo cual está relacionado con los tiempos para la entrega de las retroalimentaciones a los compañeros, que en varios casos no se dieron dentro de los plazos requeridos, lo que dificultó que las retroalimentaciones fueran aprovechadas al máximo en términos de que 
se daba paso a la siguiente actividad sin haber retroalimentado la anterior.

\section{Consideraciones finales}

Desde el análisis de todo el proceso, las actividades y los cuestionarios de indagación, se evidencia que los diferentes elementos curriculares permitieron abordar las CSC como una estrategia didáctica ya que los participantes reconocieron que las CSC involucran un asunto controvertido, con discusiones que se fortalecen por medio del debate y la interacción social, y asuntos en los que se involucran las consideraciones éticas y juicios morales acerca de temas científicos.

Por ende, es importante resaltar que abordar las CSC es una estrategia que ayuda a reflejar procesos valorativos de la ciencia para la construcción cultural y para poder hacer una evaluación sensata de sus implicaciones, con el fin de no divorciar la ciencia con su función social en términos de la formación ciudadana. Se comprende igualmente, que la finalidad de abordar una CSC tampoco es alcanzar una postura unánime en un grupo de estudiantes, sino fortalecer las posturas diversas y el pensamiento crítico desde la argumentación. Estos aspectos son promovidos también en la comunidad virtual del proyecto Engage (Unión Europea, s.f.), la que además ofrece una gran variedad de material libre para que los profesores aborden controversias de interés y contexto mundial, por lo que se recomienda ampliamente visitarla.

En cuanto a las consideraciones técnicas y tecnológicas para el desarrollo del MOOC, afortunadamente existen plataformas de acceso libre y gratuito como la que se usó en este caso; sin embargo para el desarrollo de un curso con más participantes, este tipo de plataformas pueden ser limitantes para llevar un proceso que cumpla con las expectativas tanto de los estudiantes como de los profesores que diseñan el MOOC. De ahí, que existan plataformas especializadas para el desarrollo de este tipo de cursos, que cuentan con herramientas que automatizan varios aspectos relacionados con la gestión el MOOC como por ejemplo el envío aleatorio de las actividades P2P a los diferentes participantes, y el envío correspondiente de las retroalimentaciones. Sin embargo, el acceso a estas plataformas no es fácil y se ha desarrollado todo un modelo financiero alrededor de ello, que depende fundamentalmente del modelo de financiación de las instituciones que ofrecen las plataformas o los 
MOOC (Daniel y Vásquez, 2015). "Por ejemplo, las universidades europeas se financian principalmente a través de fondos públicos y surge el dilema de si es lícito que una universidad pública ofrezca cursos gratuitos y más si cabe en estos tiempos de crisis económica" (Daniel y Vásquez, 2015), lo que en efecto significa un desafío para que las universidades públicas accedan a estas plataformas.

Esta experiencia pone sobre la mesa que existe una necesidad de la Universidad Pedagógica Nacional en ampliar la formación de los profesores para pensar en términos de educación virtual a distancia, no como un traslado de los contenidos de la educación presencial a los medios digitales, sino una formación que abarque a profundidad todas las reflexiones pedagógicas y didácticas que ello implica (uso de TIC, creación de recursos educativos abiertos, currículo, conectivismo, metodologías, evaluación virtual, roles entre estudiante-profesor, entre otras), teniendo en cuenta que implica oportunidades de hacer presencia en y de fortalecimiento de la divulgación y apropiación social del conocimiento que se genera en los grupos de investigación de la Universidad (Barrantes, Beltrán, \& Pérez 2016).

A pesar de no ser un curso con cientos de estudiantes, esto posibilitó el manejo de la dicotomía de amplitud vs profundidad en el acompañamiento a los estudiantes, teniendo en cuenta que esta situación facilitó el proceso de heteroevaluación y un seguimiento más certero sobre sus procesos.

No obstante de la característica de ser un MOOC abierto pero no masivo (Baggaley, 2013), de tratarse de una prueba piloto y del debate tecnológico y económico; esto no impidió el desarrollo del curso en términos educativos y el análisis de sus aportes y dificultades.

Con respecto a esta experiencia, es recomendable que las investigaciones acerca de $\mathrm{MOOC}$ se desarrollen más allá del tema cuantitativo de la participación y los resultados obtenidos sino también de la influencia de las redes sociales al proceso de aprendizaje teniendo en cuenta la interacción profesor-estudiante (Castaño, Maiz y Gara, 2015) considerando que los MOOC continúan siendo una oportunidad para que el estudiante aprenda, teniendo en cuenta que se trata de una modalidad para la educación no formal.

Continúa siendo un reto identificar los procesos y prácticas que puedan motivar a los estudiantes "merodeadores" o "catadores" (Hill, 2013) a 
ser más activos o participativos en el curso MOOC (Valverde, 2014), sin embargo esto está relacionado con la autonomía de los participantes junto a la construcción de una cultura digital en Latinoamérica (Aparicio, 2017) y una reconfiguración de lo que San Juan (2008) denomina como el habitus tecnológico o las disposiciones para el buen uso de la tecnología con el fin de sacarle el mejor provecho al uso de las TIC.

\section{Referencias bibliográficas}

Aparicio, O.Y. (2017). Las TIC como herramientas cognitivas. Revista Interamericana de Investigación, Educación y Pedagogía, RIIEP, 10(1). https://orcid.org/0000-0003-35356288

Azevedo, S. R. J. (2014). La composición histórica de la educación religiosa como componente curricular. Revista Interamericana de Investigación, Educación y Pedagogía, RIIEP, 7(1). DOI: https://doi.org/10.15332/s1657-107X.2014.0001.03

Alemán, L. (2015). Indicadores de calidad pedagógica para el diseño de un curso en línea masivo y abierto de actualización docente. Revista de Universidad y Sociedad del Conocimiento, 12(1), 104.119. doi:http://dx.doi.org/10.7238/rusc.v12i1.2260

Barrantes, H. A., Beltrán, J. E. P., \& Pérez, F. A. R. (2016). Perfil del estudiante de pregrado de la Facultad de Estudios a Distancia de la Universidad Militar Nueva Granada. Revista Interamericana de Investigación, Educación y Pedagogía, RIIEP, 9(2). DOI: https://doi.org/10.22490/25391887.1948

Baggaley, J. (2013). MOOC rampant. Distance Education, 34(3), 368-378. doi:10.1080/0 1587919.2013.835768

Castaño, C., Maiz, I., \& Gara, U. (2015). Redes sociales y aprendizaje cooperativo en un MOOC. Revista Complutense de Educación, 26(Especial), 119-139. doi:http://dx.doi. org/10.5209/rev_RCED.2015.v26.46328

Clarà, M., \& Barberà, E. (2013). Learning online: massive open online courses (MOOCs), connectivism, and cultural psychology. Distance Education, 34(1), 129-136.

de la Calle, C. V., Malaver, M. O., Gallego, J. D. M., Rodríguez, M., Flórez, J. C., Henao, C. E. \& Saldaña, R. (2014). Aportes de los doctorados de educación en ciencia, tecnología y sociedad, desde la sistematización de sus investigaciones doctorales científicas y formativas, 2000-2010. Revista Interamericana de Investigación, Educación y Pedagogía, RIIEP, 7(1). DOI: https://doi.org/10.15332/s1657-107X.2014.0001.04 
Daniel, J., \& Vásquez, E. (2015). El futuro de los MOOC: ¿aprendizaje adaptativo o modelo de negocio? RUSC. Revista de Universidad y Sociedad del Conocimiento, 12(1), 64-74. doi:http://dx.doi.org/10.7238/rusc.v12i1.2475

Franco, P., \& Santos, A. (2015). A viabilidade do Ensino de Biologia por meio do Enfoque CTS: possibilidades e desafios identificados a partir de uma proposta de estágio. En L. Martínez, D. Parga, e I. Garzón, Formación de Profesores y cuestiones sociocientificas: experiencias y desafíos en la interfaz universidad-escuela (págs. 239263). Universidad Pedagógica Nacional.

Ferreyra, H. A. (2014). Mesas Socioeducativas para la Inclusión y la Igualdad. Un programa "De todos con todos". Una experiencia en construcción. Revista Interamericana de Investigación, Educación y Pedagogía, RIIEP, 7(2). DOI: https://doi. org/10.15332/s1657-107X.2014.0002.01

Hill, P. (6 de Marzo de 2013). Emerging Student Patterns in MOOCs: A Graphical View. Obtenido de e-Literate: http://mfeldstein.com/emerging_student_patterns_in_moocs_ graphical_view/

Konieczny, P. (2015). Lorenzo García Aretio: bases, mediaciones y futuro de la educación a distancia en la sociedad digital. Revista Interamericana de Investigación, Educación y Pedagogía, RIIEP, 8(1). DOI: https://doi.org/10.15332/s1657107X.2015.0001.08

Liu, M., Kang, J., Mengwen, C., Lim, M., Ko, Y., Myers, R., \& Schmitz, A. (2014). Understanding MOOCs as an Emerging Online Learning Tool: Perspectives from the Students. The American Journal of Distance Education, 28, 147-159.

Manjarrés, M., Mejía, M., Bravo, A., Boada, M., \&; Jiménez, G. (2007). La pregunta como punto de partida y estrategia metodológica. Bogota: Colciencias.

Martínez, L. (2014). Cuestiones sociocientíficas en la formación de profesores de ciencias: aportes y desafíos. TED, 77-94.

Martínez, L., \& Pacheco, W. (2012). Contribuições e dificuldades da abordagem de questões sociocientíficas na prática de professores de ciências. Educação e Pesquisa, 38(3), 727-741.

Martínez, L., \& Parga, D. (2013). Discurso ético y ambiental sobre cuestiones sociocientíficas: Aportes para la formación del profesorado. Bogotá, Colombia: Universidad Pedagógica Nacional.

Mejía, M. (2012). Sistematización. Una forma de investigar las prácticas y de producción de saberes y conocimientos. La Paz: Viceministerio de educación alternativa y especial.

Pérez, T. H. P. (2013). Aproximaciones al estado de la cuestión de la investigación en educación y derechos humanos. Revista Interamericana de Investigación, Educación y Pedagogía, RIIEP, 6(1). DOI: https://doi.org/10.15332/s1657-107X.2013.0001.05 
Pérez, T. H. (2014). Colombia: de la educación en emergencia hacia una educación para el posconflicto y la paz. Revista Interamericana de Investigación, Educación y Pedagogía, RIIEP, 7(2). DOI: https://doi.org/10.15332/s1657-107X.2014.0002.06

Reis, P. (2014). Acción Socio-Política sobre Cuestiones Socio-Científicas:

Reconstruyendo la Formación Docente y el Currículo. Uni-pluri/versidad, 14(2), 16-26. Obtenido de http://aprendeenlinea.udea.edu.co/revistas/index.php/unip/article/ view/20051/16941

Sánchez, M., \& Prendes, M. (Enero de 2015). Más allá de las pruebas objetivas y la evaluación por pares: alternativas de evaluación en los MOOC. Revista de Universidad y Sociedad del Conocimiento, 12(1), 119-131.

San Juan, I. (2008). Cultura digital y reconfiguración del habitus tecnológico.

Reflexiones teóricas y filosóficas. Razón y Palabra, 13(64). Obtenido de http://www. redalyc.org/articulo.oa?id=199520727011

Valles, M. (1999). Técnicas cualitativas de investigación social. Madrid: Síntesis S.A.

Valverde, J. (2014). MOOCs: Una visión crítica desde las ciencias de la educación. Profesorado - Revista de currículum y formación del profesorado, 18(1), 93-111. Obtenido de http://www.ugr.es/local/recfpro/rev181ART6.pdf

Viejo, C. M., Cabezas, I. L., \& Martínez, M. D. J. I. (2013). Las redes de académicas en la docencia universitaria. Revista Interamericana de Investigación, Educación y Pedagogía, RIIEP, 6(2). DOI: https://doi.org/10.15332/s1657-107X.2013.0002.03

Zeidler, D. L. (2003). The Role of Moral Reasoning on Socioscientific Issues and Discourse in Science Education. Dordrecht/Boston/London: Kluwer Academic Publishers. 